\title{
Optical binding of electrically small magnetodielectric particles
}

\author{
Kesava Jay, ${ }^{1}$ Patrick C. Chaumet, ${ }^{2}$ T. N. Langtry, ${ }^{1}$ and Adel Rahmani ${ }^{1}$ \\ ${ }^{1}$ Department of Mathematical Sciences, University of Technology Sydney, \\ Broadway NSW 2007, Australia \\ ${ }^{2}$ Institut Fresnel (UMR 6133), Faculté de Saint Jérôme, Av. Escadrille Normandie-Niemen \\ 13397 Marseille cedex 20 France \\ Adel.Rahmani@uts . edu . au
}

\begin{abstract}
An ensemble of spherical particles with arbitrary dielectric permittivity and magnetic permeability was considered in the dipole approximation. Each particle was described by complex electric and magnetic polarizabilities. A computational approach based on the coupled dipole method, also called the discrete dipole approximation, was used to derive the optical force experienced by each particle due to an incident electromänetic field and the fields scattered by all other particles. This approach is general and can handle material dispersion and losses. In order to illustrate this approach, we studied the case of two spherical particles separated by a distance $d$, and illuminated by an incident plane wave whose wave vector is normal to the axis of the particles. We computed the optical force experienced by each particle in the direction of the beam (radiation pressure), and perpendicular to the beam (optical binding) for particles with positive and negative refractive indices. We also considered the effect of material losses.
\end{abstract}

\section{INTRODUCTION}

Radiation pressure is perhaps the most commonly known manifestation of optical forces [1-5]. When an electromagnetic wave is reflected off the surface of an object, momentum is imparted to the object such that the total momentum of the system (wave+object) is conserved. In the general case, however, optical forces are not govemed simply by reflections, but also depend on whether the fields vary rapidly in space (gradient force) and/or whether the materials are lossy [6]. When two or more objects are present, the multiple scattering between the objects can, under certain conditions, lead to optically bound states. This is often referred to as optical binding, and the first experimental demonstration was carried out by Burns et al . on a system of two plastic spheres in water, for which a series of bound states was observed [7]: More recentiy extended optically bound chains of microparticle were created with the use of counterpropagating waves [8]. Optical binding has been studied theoretically for two dielectric particles, in free space or put upon a flat dielectric substrate [9], for systems of metallic nanoparticles [10], and more recently, between a large number of dielectric spheres [11]. The interest in optical binding lies in its ability to create extended structure whose geometric, and by extension, electromagnetic characteristics can be tailored by an incident field. Optical binding has traditionally been studied with nonmagnetic particles, in which case the magnetic part of the electromagnetic field can be ignored, however, for magnetic and metamaterials, magnetic contributions to the force must be accounted for. This article addresses the optical force between two magnetodielectric particles, in the dipole approximation. 


\section{OPTICAL FORCE ON AN ELECTRICALLY SMALL MAGNETODIELECTRIC SPHERE}

When an arbitrary electromagnetic wave $\left\{\mathbf{E}_{0}(\mathbf{r}, \omega), \mathbf{H}_{0}(\mathbf{r}, \omega)\right\}$ (where $\omega$ is the angular frequency) impinges on a particle with permittivity $\epsilon$ and permeability $\mu$, it generates a force that results from the exchānge of momentum between the particle and the fields. Assuming a time harmonic dependence (i.e., $e^{-i \omega t}$ ) and omitting the dependence of the fields on $\omega$, the time averaged total force $\mathbf{F}$ on the particle can be written as [12]:

$$
\mathbf{F}=\frac{1}{8 \pi} \operatorname{Re}\left[\int_{S}\left[(\mathbf{E}(\mathbf{r}) \cdot \mathbf{n}) \mathbf{E}^{*}(\mathbf{r})+(\mathbf{H}(\mathbf{r}) \cdot \mathbf{n}) \mathbf{H}^{*}(\mathbf{r})-\frac{1}{2}\left(\left[\left.\mathbf{E}(\mathbf{r})\right|^{2}+|\mathbf{H}(\mathbf{r})|^{2}\right) \mathbf{n}\right] \mathrm{d} S\right]\right.
$$

where $S$ is a surface enclosing the particle, the unit vector $\mathbf{n}$ defines the local outward normal to $S$, * denotes the complex conjugate, and Re represents the real part of a complex number. $\mathbf{E}(\mathbf{r})$ and $\mathbf{H}(\mathbf{r})$ are the total fields, i.e. the sum of the incident $\mathrm{EM}$ fields $\left\{\mathbf{E}_{0}(\mathbf{r}), \mathbf{H}_{0}(\mathbf{r})\right\}$ and the $\mathrm{EM}$ fields scattered by the object $\left\{\mathrm{E}_{d}(\mathbf{r}), \mathbf{H}_{d}(\mathbf{r})\right\}$. Let $\mathbf{p}$ and $\mathbf{m}$ be the electric and magnetic dipoles induced by the incident EM wave, and let $\hat{\mathrm{r}}$ be the unit vector in the direction of $r$. The fields scattered by the object are $[13,14]$ :

$$
\begin{aligned}
\mathbf{E}_{d}(\mathbf{r})= & e^{i k r}\left\{[3 \hat{\mathbf{r}}(\hat{\mathbf{r}} \cdot \mathbf{p})-\mathbf{p}]\left(\frac{1}{r^{3}}-\frac{i k}{r^{2}}\right)+\frac{k^{2}}{r}(\hat{\mathbf{r}} \times \mathbf{p}) \times \hat{\mathbf{r}}\right. \\
& \left.-k^{2}(\hat{\mathbf{r}} \times \mathbf{m})\left(\frac{1}{r}+\frac{i}{k r^{2}}\right)\right\} \\
= & \mathbf{T}^{\mathrm{ee}} \cdot \mathbf{p}+\mathrm{T}^{\mathrm{em}} \cdot \mathbf{m} \\
\mathbf{H}_{d}(\mathbf{r})= & e^{i k r}\left\{[3 \hat{\mathbf{r}}(\hat{\mathbf{r}} \cdot \mathbf{m})-\mathbf{m}]\left(\frac{1}{r^{3}}-\frac{i k}{r^{2}}\right)+\frac{k^{2}}{r}(\hat{\mathbf{r}} \times \mathbf{m}) \times \hat{\mathbf{r}}\right. \\
& \left.+k^{2}(\hat{\mathbf{r}} \times \mathbf{p})\left(\frac{1}{r}+\frac{i}{k r^{2}}\right)\right\} \\
= & \mathbf{T}^{\mathrm{me}} \cdot \mathbf{p}+\mathbf{T}^{\mathrm{mm}} \cdot \mathbf{m},
\end{aligned}
$$

where $\mathrm{k}$ is the wave vector. The quantities $\mathbf{T}$ are field susceptibility tensors [15] and the superscripts relate to the electric or magnetic nature of the field and the source. Notice that in cgs the field susceptibility tensors satisfy $\mathrm{T}^{\mathrm{ee}}=\mathrm{T}^{\mathrm{mm}}$ and $\mathrm{T}^{\mathrm{em}}=-\mathrm{T}^{\mathrm{me}}$. After some manipulations, the total force experienced by the particle can be written as [13]:

$$
F^{i}=\frac{1}{2} \operatorname{Re}\left[p^{j} \partial^{i} E_{0}^{* j}+m^{j} \partial^{i} H_{0}^{* j}-\frac{2 k^{4}}{3} \epsilon^{i j k} p^{j} m^{* k}\right] .
$$

where $\epsilon_{i j k}$ is the Levi-Civita tensor, $\partial^{i}$ denotes partial differentiation with respect to the $i^{\text {th }}$ Cartesian component, and repeated indices are summed over. If we introduce the electric and magnetic polarizabilities of an electrically small sphere of radius $a$, we have $\mathrm{p}=\alpha^{\mathrm{e}} \mathrm{E}_{0}$ and $\mathrm{m}=\alpha \stackrel{\mathrm{m}}{=} \mathbf{H}_{0}$ with the polarizabilities written as:

$$
\alpha^{\mathrm{e}}=\alpha_{0}^{\mathrm{e}} /\left(1-\frac{2}{3} i k^{3} \alpha_{0}^{\mathrm{e}}\right) \quad \text { and } \quad \alpha^{\mathrm{m}}=\alpha_{0}^{\mathrm{m}} /\left(1-\frac{2}{3} i k^{3} \alpha_{0}^{\mathrm{m}}\right)
$$

where

$$
\alpha_{0}^{e}=a^{3} \frac{\epsilon-1}{\epsilon+2} \quad \text { and } \quad \alpha_{0}^{\mathrm{m}}=a^{3} \frac{\mu-1}{\mu+2}
$$

and the $i^{\text {th }}$ component of the total force can be written as:

$$
F^{i}=\frac{1}{2} \operatorname{Re}\left[\alpha^{\mathrm{e}} E_{0}^{j} \partial^{i} E_{0}^{* j}+\alpha^{\mathrm{m}} H_{0}^{j} \partial^{i} H_{0}^{* j}-\frac{2 k^{4}}{3} \epsilon^{i j k} \alpha^{\mathrm{e}} E_{0}^{j}\left(\alpha^{\mathrm{m}} H_{0}^{k}\right)^{*}\right] .
$$


Compared to the case of a single electric dipole [16], we now also have a contribution to the optical force that comes from the magnetic dipole, as well as a self interaction term involving the electric and magnetic dipole moments.

\section{OPTICAL FORCE IN A SYSTEM OF $N$ MAGNETODIELECTRIC PARTICLES}

Consider $N$ particles. The local fields at particle $l$ is the sum of the incident field and the field scattered by the other spheres and then can be written as [17]:

$$
\begin{aligned}
\mathbf{E}_{l} & =\mathbf{E}_{0 l}+\sum_{n=1}^{N}\left[\mathbf{T}_{l n}^{\mathrm{ee}} \alpha_{n}^{\mathrm{e}} \mathbf{E}_{n}+\mathbf{T}_{l n}^{\mathrm{em}} \alpha_{n}^{\mathrm{m}} \mathbf{H}_{n}\right] \\
\mathbf{H}_{l} & =\mathbf{H}_{0 l}+\sum_{n=1}^{N}\left[\mathbf{T}_{l n}^{\mathrm{me}} \alpha_{n}^{\mathrm{e}} \mathbf{E}_{n}+\mathbf{T}_{l n}^{\mathrm{mm}} \alpha_{n}^{\mathrm{m}} \mathbf{H}_{n}\right],
\end{aligned}
$$

where the terms $\mathbf{T}$ are the field susceptibility tensors defined in Eqs. (2)-(4). If we write the equations for the local fields for all $N$ particles, we get a linear system of size $6 N \times 6 N$ which can be solved ùsing, for instance, iterative methods [18]. We now have the fields, however we also need their spatial derivatives to derive the optical forces. The spatial derivatives of the fields at particle $l$ are obtained through:

$$
\begin{aligned}
& \nabla \mathbf{E}_{l}=\nabla \mathbf{E}_{0 l}+\sum_{n=1}^{N}\left[\nabla \mathbf{T}_{l n}^{e \mathrm{e}} \alpha_{n}^{\mathrm{e}} \mathbf{E}_{n}+\nabla \mathbf{T}_{l n}^{\mathrm{em}} \alpha_{n}^{\mathrm{m}} \mathbf{H}_{n}\right] \\
& \nabla \mathbf{H}_{l}=\nabla \mathbf{H}_{0 l}+\sum_{n=l}^{N}\left[\nabla \mathrm{T}_{l n}^{\mathrm{me}} \alpha_{n}^{\mathrm{e}} \mathbf{E}_{n}^{-}+\nabla \mathrm{T}_{l n}^{\mathrm{mm}} \alpha_{n}^{\mathrm{m}} \mathbf{H}_{n}\right]
\end{aligned}
$$

From this point, the force on each particle is derived using Eq. (9). Note that this approach can also be used to derive electromagnetic forces on an arbitrary object (dielectric object in presence of a substrate $[19,20]$, or close to a photonic crystal [21], metallic object [22] or magnetic object [13]).

\section{ELECTROMAGNETIC BINDING BETWEEN 2 MAGNETODIELECTRIC PARTICLES}

To illustrate our approach we consider 2 spheres (radius $\lambda / 50$ ) illuminated by a plane wave traveling in the $z$ direction and we calculate the force experienced by each particle as a function of the distance between them (normalized to the wavelength of the incident wave in the subsequent plots) (Fig. 1). We consider two polarization states of the incident wave. In $p$ polarization the electric field of the incident wave is polarized along the direction $(x)$ defined by the two particles. In $s$ polarization, the electric field of the incident wave is polarized along the $y$ direction (and the magnetic field is along the $x$ axis).

On the plots below, the black and red curve are associated with the left and right particle, respectively. This means that when the force along $x$ is positive for the black curve and negative for the red curve, the particles are attracted toward each other.

In Fig. 2 we start by considering the case of two identical particles. In Figs. 2(a)-(b) we have two non magnetic particles with $\epsilon=2$ and $\mu=1$ for the two polarizations of the incident wave (solid curves). We also consider the effect of losses by adding an imaginary part of 0.5 to the permittivity (dashed curves). For both polarization we observe the usual oscillations of the force that result from the interference between the ficlds scattered by the particles (rctardation effect) as the distance between them is varied. However, we also notice a significant difference 


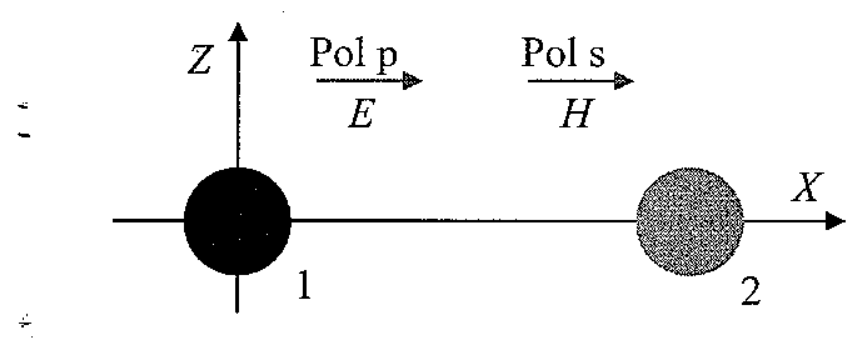

Fig. 1. Schematic of the configuration

in the near field (i.e., short range) behavior of the force for the two polarizations. For the $p$ polarization, the short range optical force is attractive whereas it is repulsive for the $s$ polarization. We also note that, in the present case, the overall effect of losses is to only increase slightly the magnitude of the force. Similarly, the magnitude of the force along $z$ (not show) increases when losses are introduced. This increase is the result of a scattering contribution to the force (radiation pressure) due to the material absorption [6].

.. In Figs:-2(c)-(d) we consider two particles with $\epsilon=\mu=2$ (solid curves) or $\epsilon=\mu=$ $2+0.5 i$ (dashed curves). This time we notice that the short range interaction leads to an attractive (binding) force for both polarizations. This result is quite different from the case of two dielectric spheres for which we get antibonding states and bonding states in the near field depending on the polarization of the incident field [9]. In fact, the evolution of the force experienced by the particles versus their separation is identical for $s$ and $p$ polarizations. This symmetry is a consequence of the symmetry of the optical constants of the two particles. Indeed, when we exchange the polarizations it is equivalent to exchanging the roles of $\epsilon$ and $\mu$ in the expression of the force. Since we have $\epsilon=\mu$, the force for the two polarizations are identical. With this in mind, when the particles are identical and we have $\epsilon=\mu$ we will plot the $x$ component of the force for one polarization only, since the other polarization would give the same result. We also note that similar to the previous case, the introduction of losses leads to an overall increase of the magnitude of the force.

In Figs. 3(a)-(b) we consider two particles with $\epsilon=\mu=-2$ (solid curves). Note that these values correspond to the "plasmon resonance" for both the electric and the magnetic polarizabilities. Incidentally, we note that Lakhtakia has recently shown that mixing of electric and magnetic dipole contributions occurs for low $k a$ spheres [23]. We also consider the effect of losses. However, because the polarizabilities of the particles exhibit a resonant behaviour when $\epsilon$ and/or $\mu$ are near -2 , we can expect that the influence of losses on the force will be stronger than in the previous, non resonant cases. To illustrate this, we consider two level of losses: $\epsilon=\mu=-2+0.01 i$ (dashed curves) and $\epsilon=\mu=-2+0.1 i$ (dashed dotted curves)

We plot in Fig. 3(a) the $x$ component of the force. We first note that in the lossless case, due to the aforementioned resonances the magnitude of the force is several orders of magnitudes larger than in the previous case $(\epsilon=\mu,=, 2)$. We also observe that the force between the particles is strongly repulsive for a separation of about 0.3 wavelengths and it is also repulsive, albeit in a smaller way, at very short distances. To find whether stable binding configurations exist, one would have to look at the trapping potential for a given incident power of the trapping laser and compare that to the effect of Brownian motion when the particles are in a fluid. This is beyond the scope of this work.

An interesting effect is illustrated in Fig. 3(b) where we plot the $z$ component of the force (radiation pressure). We observe that when the particles are far apart they both experience a strong radiation pressure (the force along the $z$ direction is the same for the two particles due to the symmetry of our configuration). However, as the distance between the two particles is reduced the $z$ component of the force decreases rapidly. This is due to the resonant effect we 

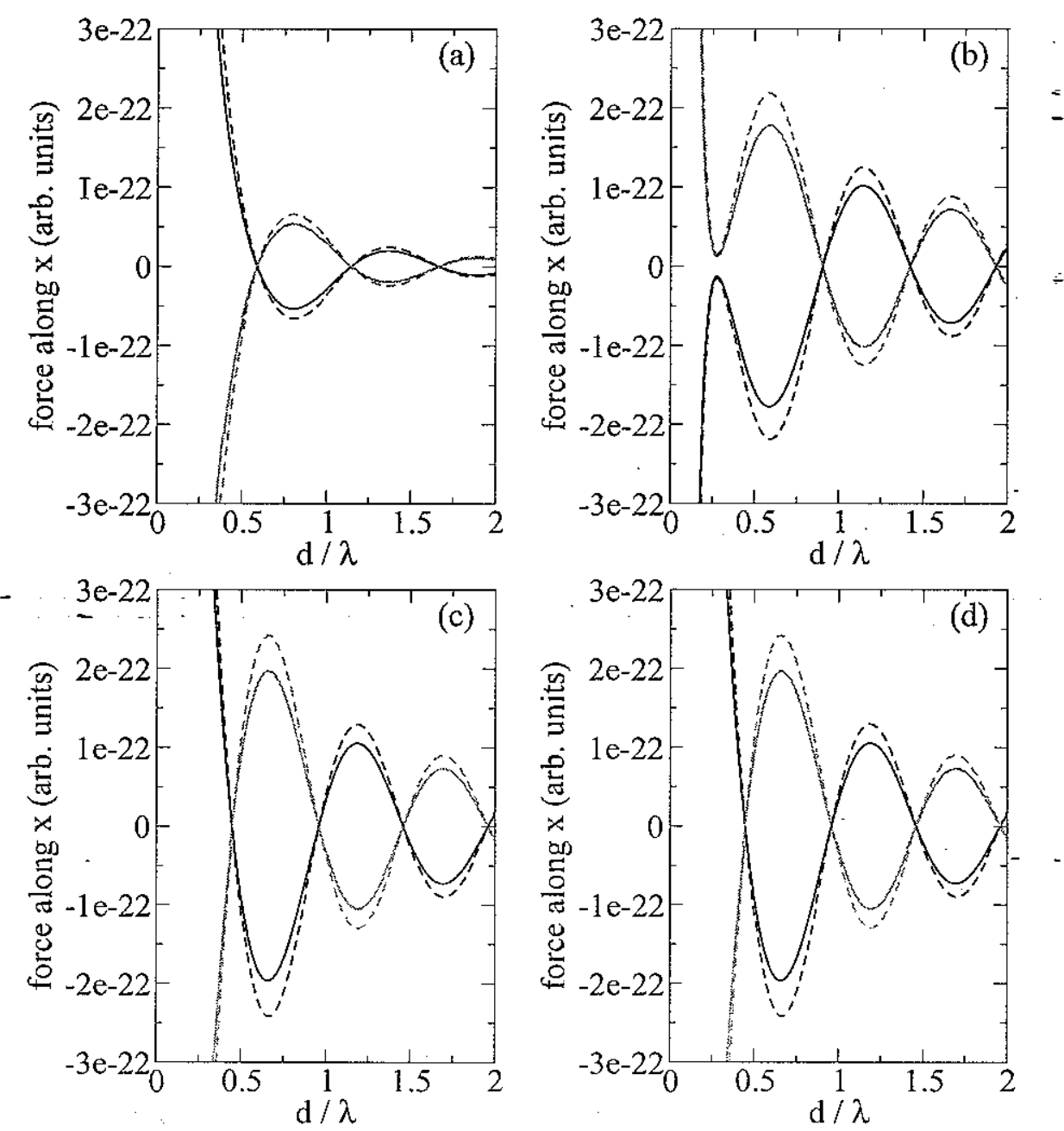

Fig. 2. $x$ component of the force on left (black) and right (red) particles as a function of the separation along $x$. The particles are identical. $\epsilon=2, \mu=1$ (solid curves); $\epsilon=2+0.5 i, \mu=1$ (dashed curves), (a): $p$ polarization, (b): s polarization ; $\epsilon=2, \mu=2$ (solid curves); $\epsilon=$ $2+0.5 i, \mu=2+0.5 i$ (dashed curves), (c): $p$ polarization, (d): $s$ polarization.

mentioned previously. One way to think about the radiation pressure effect is to consider not the physical size of the particle, but its effective size (extinction cross section [1]). When the particles are far apart they behave pretty much like two isolated particles with a large effective size and therefore a large radiation pressure. When the particles are close to each other, their mutual coupling reduces the effective size of the the system of two particles resulting in a weaker radiation pressure.

We also see that whereas in the previous, non resonant cases the effect of material losses was to increase the magnitude of the force, in the present case, the presence of material loss entails a damping of the resonance of the polarizabilities of the particle which leads to an overall weaker optical force when the losses increase.

In Figs. 3(c)-(d) we consider two particles with $\epsilon=\mu=-1$. The force along $x$ exhibit a sharp attractive (binding) behavior when the particles are brought together before switching to a strongly repulsive force at very close range. Unlike in the previous (resonant) case, the force in 

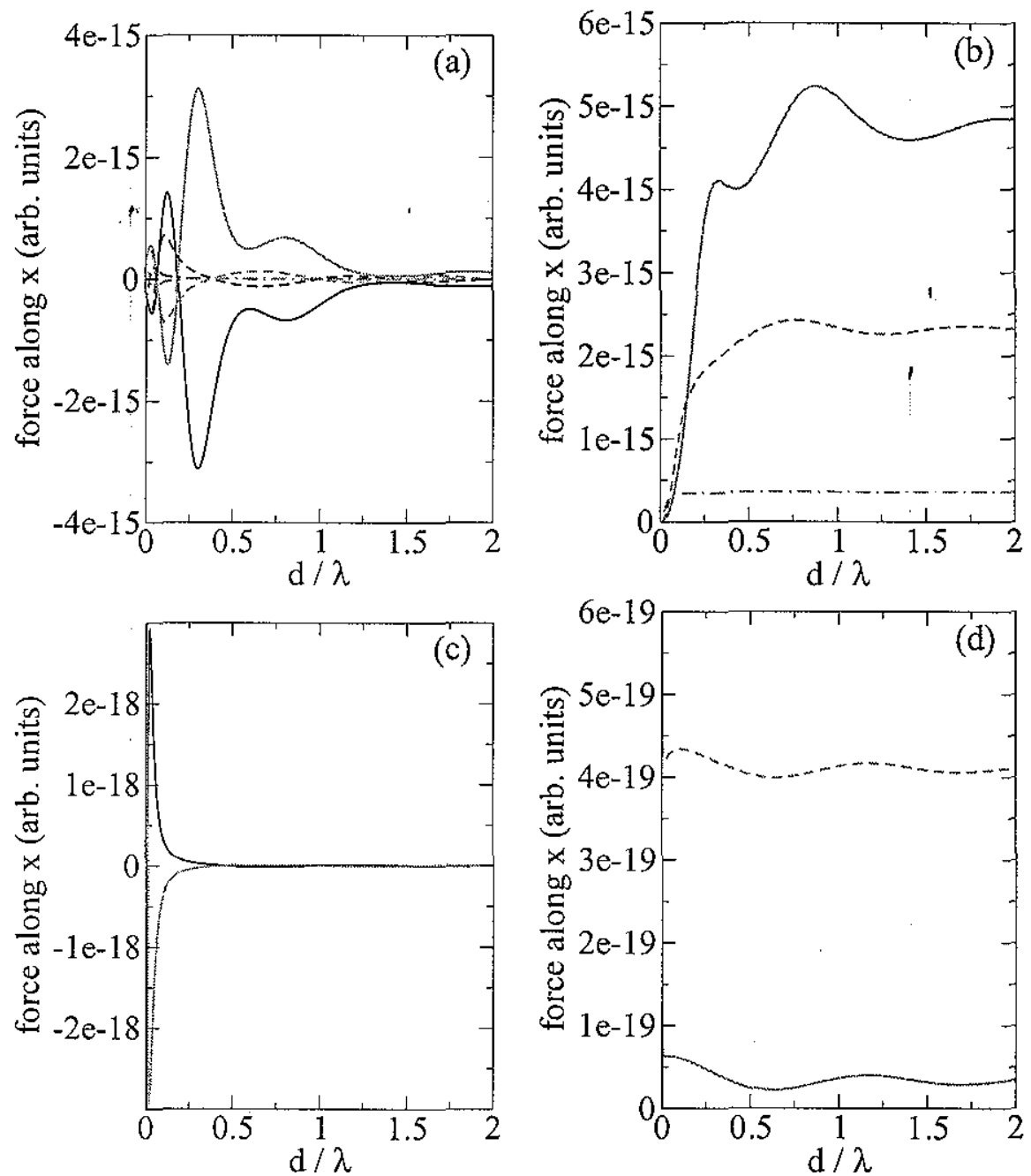

Fig. 3. Electromagnetic force ( $s$ polarization) on left (black) and right (red) particles as a function of the separation along $x$. The particles are identical. $\epsilon=-2, \mu=-2$ (solid curves); $\epsilon=\mu=-2+0.01 i$ (dashed curves); $\epsilon=\mu=-2+0.1 i$ (dashed dotted curves), (a): $F_{x},(b)$ : $F_{z} ; \epsilon=\mu=-1$ (solid curves); $\epsilon=\mu=-1+0.01 i$ (dashed curves), (c): $F_{x}$, (d): $F_{z}$.

the $z$ direction, in the near field regime, increases monotonically when the particles are brought together. Also, the effect of absorption (only shown for the force along $z$ ) is similar to what was observed in Fig. 2 and leads to an increase of the radiation pressure.

So far we have considered identical particles, mainly because it leads to an evolution of the optical force with the separation between the two particles that is easier to analyse. However, our approach of course still applies for particles with different optical constants. In Fig. 4 we consider the case of two particles with optical constants $\left(\epsilon_{1}=-2, \mu_{1}=-1\right)$ (particle on the left) and ( $\left.c_{2}=-3, \mu_{2}=-2\right)$ (particle on the right). These optical constants correspond to a resonance of the electric polarizability for the first particle, and a resonance of the magnetic polarizability for the second particle. Figures 4(a)-(b) show the $x$ and $z$ components of the force for a $p$ polarized incident plane wave. If we focus on Fig. 4(a) first, we observe the usual oscillations of the force with the distance between the particles except that, when the particles 

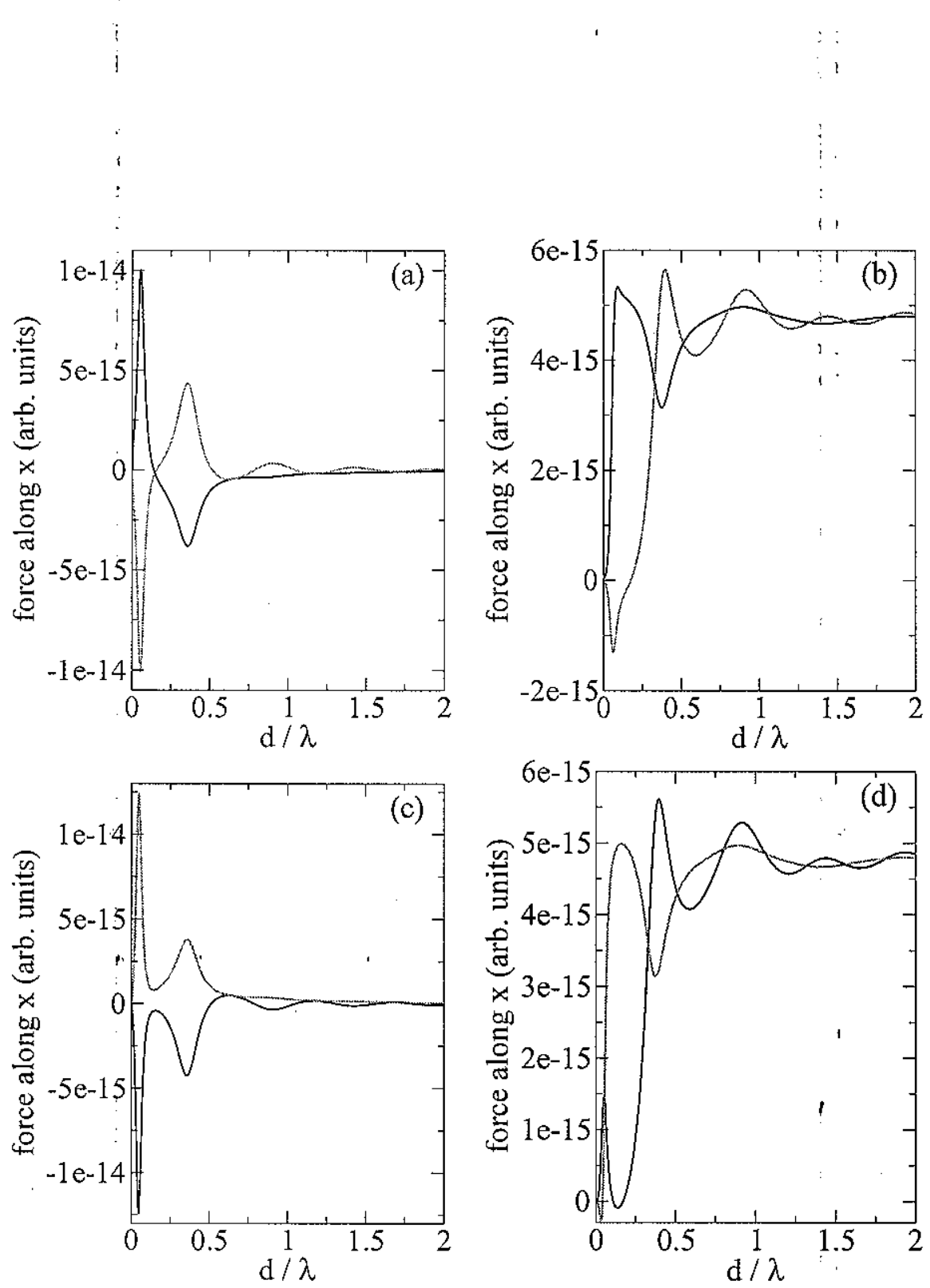

Fig. 4. Electromagnetic force on left (black) and right (red) particles as a function of the separation along $x . \epsilon_{1}=-2, \mu_{1}=-1, \epsilon_{2}=-3, \mu_{2}=-2$, (a): $F_{x}, p$ polarization, (b): $F_{z}, p$ polarization; (c): $F_{x}, s$ polarization, (d): $F_{z}^{\prime}, s$ polarization.

are far apart, the force is stronger on the second particle. This is, of course, a consequence of the asymmetry between the two particles. Similar to the case of two particles with $\epsilon=\mu=-2$ (Fig. 3), we observe a strong repulsive force for a separation of about 0.4 wavelengths, however in the present case, the $x$ component of the force reaches its maximum (or minimum for the particle on the left) at shorter distances, indicating a strong binding effect. The $z$ component of the force Fig. 4(b) exhibit quite strong oscillations. What is interesting here is that the two particles experience a different radiation pressure. This means that, were we to allow the position of the particles to evolve under the influence of the optical force, they would no longer remain aligned along the $x$ direction. In fact, we can see in Fig. 4(b) that for a small range of particle separations (between 0 and 0.25 wavelengths) the particle on the right experiences a negative force along $z$ whereas the particle on the left is púshed along the direction of propagation of the incident wave. Let us emphasize here that the negative force along $z$ on the particle on the right is not indicative of a negative radiation pressure effect (which would be expected for a particle 
in a background medium with negative $\epsilon$ and $\mu$ ). Rather, it is the result of the multiple scattering between the two non identical particles. If we switch to the $s$ polarization 4 (c)-(d): we no longer get a simple exchange of the red and black curves as we would with two identical particles. In fact, the $x$ component of the force now shows that the interaction between the two particles is always repulsive (no binding is possible) for the range of particle separation considered. On the other hand, the $z$ component of the optical force does indeed look similar to the $p$ polarization case with an exchange of the two particles, at least when the separation between the particles is larger than half a wavelength. This is a reflection of the fact that when the particles are far apart the leading contribution to the force comes from the electric dipole resonance for particle 1 and from the magnetic dipole resonance for particle 2. Accordingly, given the symmetry of the problem, outside the near field regime, the behavior of the force experienced by particle 1 in $p$ polarization is similar to that of the force experienced by particle 2 in $s$ polarization.

\section{CONCLUSION}

We have developed a general theory of electromagnetic forces for a collection of magnetodielectric particles. We have used our method to study the electromagnetic force experienced by a system of two particles, and we showed the characteristics of the force and the binding behavior depends strongly on the electric and magnetic responses of the particles. The present treatment of particles in the dipole approximation also provides a foundation for the study of optical forces in the case of more complex magnetodielectric nanostructures within the framework of the coupled dipole method (also called discrete dipole approximation) [24-27].

\section{References}

[1] C. F. Bohren and D. R. Huffman, Absorption and Scattering of Light by Small Particles, Wiley-VCH, Weinheim, Germany (2004).

[2] A. Ashkin, "Trapping of atoms by resonance radiation pressure," Phys. Rev. Lett. 40, 729732 (1978) [doi:10.1103/PhysRevLett.40.729].

[3] A. Ashkin and J. M. Dziedzic, "Observation of resonances in the radiation pressure on dielectric spheres," Phys. Rev. Lett. 38, 1351-1354 (1977) [doi:10.1103/PhysRevLett.38.1351].

[4] D. McGloin, "Optical tweezers: 20 years on," Phil. Trans. R. Soc. A 364, 3521-3537 (2006) [doi:10.1098/rsta.2006.1891].

[5] M. Dienerowitz, M. Mazilu, and K. Dholakia, "Optical manipulation of nanoparticles: a review," J. Nanophoton. 2, 021875 (2008) [doi:10.1117/1.2992045].

[6] M. Nieto-Vesperinas, P. C. Chaumet, and A. Rahmani, "Near-field photonic forces," Phil. Trans. R. Soc. Lond. A 362, 719-737 (2004) [doi:10.1098/rsta.2003.1343]:

[7] M. M. Burns, J.-M. Fournier, and J. A. Golovchenko, "Optical binding," Phys. Rev. Lett. 63, 1233-1236 (1989) [doi:10.1103/PhysRevLett.63.1233].

[8] V. Karàsek, T. Čimžàr, O. Brzøbohatỳ, P. Zemànek, V. Garceés-Chávez, and K. Dholakia, "Long-range one-dimensional longitudinal optical binding," Phys. Rev. Lett. 101, 143601 (2008) [doi: 10.1103/PhysRevLett.101.143601].

[9] P. C. Chaumet and M. Nieto-Vesperinas, "Optical binding of particles 'with or without the presence of a flat dielectric surface," Phys. Rev. B 64, 035422 (2001) [doi:10.1103/PhysRevB.64.035422].

[10] A. S. Zelenina, R. Quidant, and M. Nieto-Vesperinas, "Enhanced optical forces between coupled resonant metal nanoparticles," Opt. Lett. 32, 1156-1158 (2007) [doi:10.1364/OL.32.001156].

[11] Z. H. Hang, J. Ng, and C. T. Chan, "Stability of extended structures stabilized by light as governed by the competition of two length scales," Phys. Rev. A 77, 063838 (2008) [doi:10.1103/PhysRevA.77.063838]. 


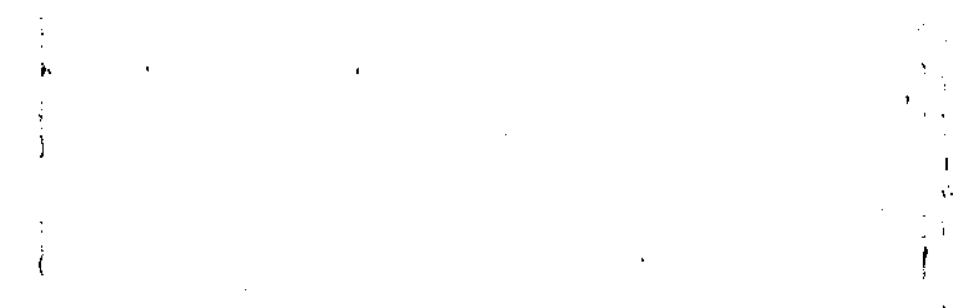

[12] J. A. Stratton, Electromagnetic Theory, McGraw-Hill, New York, USA (1941).

[13] P. C. Chaumet and A. Rahmani, "Electromagnetic force and torque on magnetic and negative-index scatterers," Opt. Exp. 17, 2224-2234 (2009) [doi:10.1364/OE.17.002224]

[14] J. D. Jackson, Classical Electrodynamics, 2nd. ed., Wiley, New York, NY, USA (1975).

[15] G. S. Agarwal, "Quantum electrodynamics in the presence of dielectrics and conductors. I Electromagnetic-field response functions and black-body fluctuations in finite geometry," Phys. Rev. A 11, 230-242 (1975) [doi:10.1103/PhysRevA.11,230].

[16] P. C. Chaumet and M. Nieto-Vesperinas, "Time-averaged total force on a dipolar sphere in an electromagnetic field," Opt. Lett. 25, 1065-1067 (2000) [doi:10.1364/OL.25.001065].

[17] A. Lakhtakia, "Strong and weak forms of the method of moments and the coupled dipole method for scattering of time-harmonic electromagnetics fields," Int. J. Mod. Phys. C 3, 583-603 (1992) [doi:10.1142/S0129183192000385]. .

[18] P. C. Chaumet and A. Rahmani, "Efficient iterative solution of the discrete dipole approximation for mag neto-dielectric scatterers " Opt. Lett. 34, 917-919 (2009) [doi: 10.1364/OL.34.000917].

[19] P. C. Chaumet, A. Rahmani, and M. Nieto-Vesperinas, "Optical trapping and manipulation of nano-objects with an apertureless probe," Phys. Rev. Lett. 88, 123601 (2002) [doi:10.1103/PhysRevLett.88.123601].

[20] P. C. Chaumet, A. Rahmani, and M. Nieto-Vesperinas, "Selective nanomanipulation using optical forces," Phys. Rev. B 66, 195405 (2002) [doi:10.1103/PhysRevB.66.195405].

[21] A. Rahmani and P. C. Chaumet, "Optical trapping near a photonic crystal," Opt. Exp. 14, 6353-6358 (2006) [doi:10.1364/OE.14,006353].

[22] P. C. Chaumet, A. Rahmani, and M. Nieto-Vesperinas, "Photonic force spectroscopy on metallic and absorbing nanoparticles," Phys. Rev. B 71, 045425 (2005) [doi:10.1103/PhysRevB.71.045425].

[23] A. Lakhtakia, "Radiation pressure efficiencies of spheres made of isotropic, achiral, passive, homogeneous, negative-phase-velocity materials," Electromagnetics 28, 346-353 (2008) [doi: 10.1080/02726340802074392].

[24] B. T. Draine, "The discrete-dipole approximation and its application to interstellar graphite grains," Astrophys. J. 333, 848-872 (1988) [doi:10.1086/166795].

[25] A. Rahmani, P. C. Chaumet, and G. W. Bryant, "Local-field correction for an interstitial impurity in a crystal," Opt. Lett. 27, 430-432 (2002) [doi:10.1364/OL.27.000430].

[26] P. C. Chaumet, A. Rahmani, and G. W. Bryant, "Generalization of the coupled dipole method to periodic structure," Phys. Rev. B 67, 165404 (2003) [doi: 10.1103/PhysRevB.67.165404].

[27] P. C. Chaumet and A. Rahmani, "Coupled-dipole method for magnetic and negative refraction materials," J. Quant. Spect. Rad. Transf. 110, 22-29 (2009) [doi: 10.1016/j.jqsrt.2008.09.004]. 